\title{
Constructing Creativity Indicator and Systematical Creativity Scale for Interior Design Students in College Education
}

\author{
Shih-Yung Liu \\ Department of Interior Design, Chung Yuan Christian University, Taoyuan, Taiwan (R.O.C.) \\ Email address: \\ 1sy@cycu.edu.tw \\ To cite this article: \\ Shih-Yung Liu. Constructing Creativity Indicator and Systematical Creativity Scale for Interior Design Students in College Education. \\ International Journal of Architecture, Arts and Applications. Vol. 2, No. 3, 2016, pp. 12-19. doi: 10.11648/j.ijaaa.20160203.11
}

Received: October 3, 2016; Accepted: October 26, 2016; Published: November 23, 2016

\begin{abstract}
This study aims to develop an assessment tool regarding creativity of college students from interior design related majors. According to Csikszentmihalyi's systems model of creativity, this study integrates affecting factors of creativity from person, society, and culture system as its research foundation. Later, it invites 17 winning students from national interior design competition and 19 teachers with lavish teaching experiences for initial interview. After interview analysis, this study comes up with 156 creativity indicators. An expert meeting with 15 scholars and experts is followed to extract and narrow the 156 indicators to 130 . The study then conducts questionnaire to 30 experts with over seven years of teaching experiences using the 130 indicators. Analysis from expert questionnaire shows that 124 indicators are filtered and selected for the final creativity assessment tool. The study results also point out that the top three dimensions that affect students' creativity are ability, thinking, and personality accordingly, while the bottom three are family, student club, and motivation. Regarding the top three influential indicators of creativity are nimble usage of knowledge, passionate about innovation, and love to image. The results of this study can be used as references for the development of creativity scale and the implement of school creativity teaching.
\end{abstract}

Keywords: Interior Design, Indicator, Systematical Creativity, Creativity Scale

\section{Introduction}

Creativity is the dominating force of design competitiveness, especially when every country sets creative industry as its competition niche. Taiwan aims to mark creativity as the foundation of national competitiveness, thus, it lists creative industry on its national development priority. University is a nation's cradle of high-end specialty. University education has gigantic influence on professional competitiveness regarding all career fields, as the cultivation of creativity plays an imperative role among all. Regarding interior design specialty, it is extremely vigorous to inspire, cultivate, and promote students' professional creativity. It would be beneficial to curriculum context and implementation if a framework of reliability and validity with systematical creativity indicator is established based on outstanding students' academic performance.

When it comes to design industry, creative genius relates to function and pleasure determines the value and popularity of one's work. Nevertheless, how is creativity originated? What does it take to gain better creativity? What affects creativity? Unfortunately, these essential questions to design industry have never been taken seriously. And it points out the importance of this study.

Academic research studies concerning creativity has accumulated considerable amount worldwide. Two principal research directions are: (1) Human characteristics oriented, i.e., mental intelligence, knowledge, perception skills, personality, and motivation. (2) Environmental element oriented, i.e., natural environment, family environment, school or working environment, career filed, and culture [1], [2], [3], [4]. Recently, scholars are gradually paying attention to the impacts to personal creativity from diverse perspectives to explore the combination of multi-factor, including culture, society, organization, team, school, peers, family, and environment [5], [6], [7], [8], [3]. Csikszentmihalyi proposes the flow of creativity that includes personal and environmental factors is considered the 
chef-d'oeuvre [9]. Systematical creativity considers creativity as a gestalt. It is difficult to explain creativity on the individual aspects, including personality, motivation, thinking, or creation. Creative work often needs approval of others, while creative thinking and resource are influenced by time and culture. This study adopts Csikszentmihalyi's systems model of creativity to discover the systematic creativity indicator of interior design. The purposes of this study are: (1) Analyzing the creating process of interior design major students to initiate an indicator system of systematic creativity. (2) Constructing a creativity scale of systematic creativity of interior design major students to discuss important indicator items that affect their creativity. The study results can be used to establish creativity scale as well as to cultivate students' creativity among interior design related academic majors.

\section{Literature Reviews}

\subsection{Definition of Creativity}

In 1950, the president of American Psychological Association, Dr. J. P. Guilford urges more devotion of creativity research from the academy field. The development of creativity research has been rising over five decades, especially in the recent 20 to 30 years, cultivation of student's creativity has become the education trend and focus worldwide. Relevant academic research and literatures are plentiful and versatile. However, it remains a controversial topic to define creativity and to assess it. Educators and psychologists see it differently. From the ability to invent, divergent thinking or productive thinking, to imagination or dual association ability, creativity can be anything and everything. Some believe that novelty, uniqueness, value, transformation, and exquisites are essence of creativity. Some consider creativity as the ability to solve problems effectively, which includes fluency, flexibility, individuality, redefinition, elaboration, expression, productivity, originality, and sensitivity. It can also be the ability to unite or connect elements into a new relation [10].

Chen [11] combines viewpoints from Runco et al [12] and sorts out the following 25 aspects of creative personality: inner motivation, endurance, perseverance, enjoy working, discipline and responsibility, stamina, enthusiasm, diligence, inquisitiveness doubtful to hypothesis, avoid fixed perception, attentiveness to novelty, innovation, distinctiveness, imagination, comprehension, flexibility, mental image, advantageous, courageous, fabrication, curiosity, vigilance, independence, and challenge the traditions. Creativity is indeed a complex concept, while the interpretation to it vary from viewpoints and research orientation. Even Torrance, who spent most of his life working on creativity argues that creativity is hard to clearly defined [13]. These diverse and versatile perspectives reflect how complex creativity is. To define creativity based on the particular aspect one wishes to find out might be an appropriate and practical approach.

\subsection{Systematical Creativity}

This study adopts Csikszentmihalyi's systems model of creativity [2], [14]. Csikszentmihalyi points out that creativity is composed of three elements, including domain, field, and person [9]. Csikszentmihalyi also argues that creativity does not always result from personal characteristics, much of it depends on acceptance regarding its creation by the domain gatekeeper to be counted in its field. A person would not own the distinctive creativity if he/she is not edified by the domain. Systems model of creativity integrates personal and environmental elements, which strongly believes that creativity results from the interaction of three main systems, include domain (cultural system), field (social system), and person (personal system) (Figure 1).

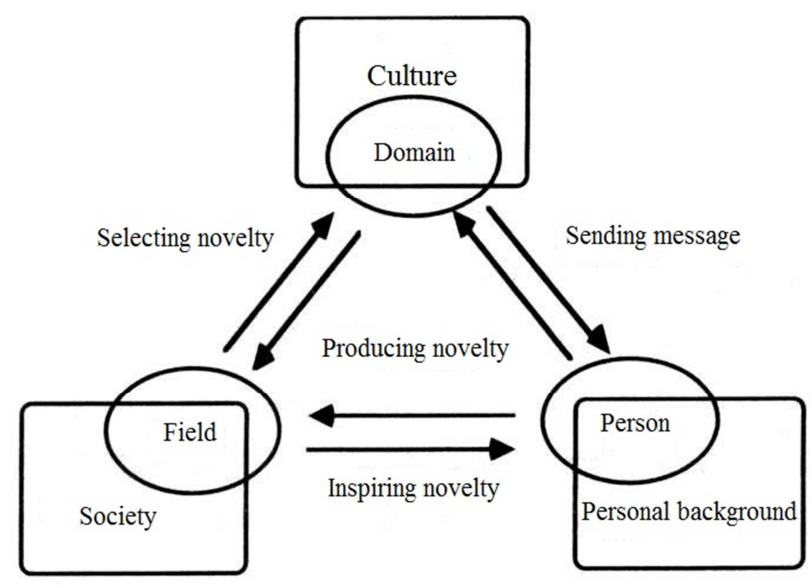

Figure 1. Systems model of creativity by Csikszentmihalyi [15].

According to Csikszentmihalyi, creativity is domainrelated, different domains generate different creativities. Though different domains share the common "general creativity", different specialty belongs to its own unique domain, where "distinctive creativity" exists in. Csikszentmihalyi argues that creativity does not always result from personal characteristics, much of it depends on acceptance regarding its creation by the domain gatekeeper. A person would not own the distinctive creativity if he/she is not edified by the domain. Creativity would only appear in the existing filed. That is to say, if a person has never been trained or edified by professional domain and knowledge, no professional creativity would be generated. Gardner points out that the extensive and influential creative response is surely connected to certain domain, involving in various skills, wide-ranging knowledge, and a significant professional training period [16]. To interior design, creativity shares common "general creativity" with other domains, while it retains its "distinctive creativity". If students wish to have extensive and influential creative responses, they must undergo a vital professional training period to cultivate and nourish the unique knowledge and skills. Therefore, one should fully understand the specialty and uniqueness of certain field before evaluating interior 
design students' professional creativity and their creations' creative level.

Csikszentmihalyi's systems model of creativity still remains as conceptual. It has not been experimented or examined yet. There is no relative research study that adopts comprehensive and systematic viewpoints so far.

\subsection{Creativity Assessment}

Creativity is an abstract potential concept. Different theory followers use different research methods to interpret creativity. Mayer sorts out six approaches of creativity research. 1. Psychometric approach sees creativity as a mental attribute. 2. Experimental approach considers it as a cognitive process. 3. Biographical approach believes individual creativity is distinctive. 4 . Biological approach argues that creativity is a brain activity. 5. Computational approach takes creativity as a mental calculation. 6. Contextual approach focuses on social, cultural, and evolutionary context [17].

Chen categorizes creativity indicator regarding ability into six categories. 1. Professional skills (professional capacity, skills, and research and development). 2 . Integration skills (well-organized, and proposal writing skills). 3. Sensitive perception (sharp observation and sensitive of marketing). 4. Responsive ability (flexibility, responsive reaction, and innovation skills). 5. Communication skills (expressive, communicating, and negotiable skills). 6. Implementation skills (execution and operation). [11]. Five categories are made regarding personality. 1. Hardworking (motivated, active, and responsible attitudes). 2. Perseverance (endurance, pressure-resisted, fear no defeat). 3. Cautiousness (serious, cautious, and thorough to work). 4. Activeness (passionate, optimistic, and confident to everything). 5. Open personality (love to interact, enjoy novelty, and outgoing personality) [18].

There are two types of creativity indicator regarding knowledge according to Chung [19]. One is related to knowledge gaining access, including getting professional knowledge from professionals, school teachers, and the internet. The other is related to knowledge gaining method, including getting knowledge by different instructions, practices, and lessons.

In addition, diverse methods result in various creativity research design and tools. This study covers from creativity process, indicator, scale, to norm establishment. Hence, the assessment comprises creativity input, creativity process, and creativity output. Regarding creativity assessment of input and process that related to individuals, divergent thinking test is used for review and analysis. The assessment tool and indicator for creativity output regarding creation is based on features of interior design work that presented to human, i.e. the combination of shape, color, and quality in three dimensional space. For a certain professional field, because it involves in different skills, various types of knowledge, and a critical specialized training period [16], the professional creativity should be established on comprehensive professional know-how and training, as well as its creativity indicator be distinctive from other professions.

\section{Research Method and Flow}

This study adopts Csikszentmihalyi's systems model of creativity, relative theories, and literatures. It firstly initiates 10 dimensions, including personality, motivation, ability, knowledge, thinking, family, school, student club, criteria, and stakeholder. Then, 17 interview questions are sorted out based on context of these 10 dimensions. Interview participants are selected from: (1) Winning students from national interior design competitions in 2016. 17 valid participants in total, coded from S-1 to S17. (2) College and university interior or space design teachers with over seven years of teaching experience. They are asked to answer questions regarding students' creativity based on their rich teaching experiences and observation. 19 valid participants, coded as A-1 to A-19. Interview record is typed, modified, and confirmed with the participants. Three experts are invited to discuss the interview results and sort out 10 dimensions with 156 creativity indicators.

An expert meeting is held with 15 experts from both interior design industry and academic field in order to extract and examine the 156 indicators from 10 dimensions. The meeting narrows them down to 130 indicators and categorizes them into a questionnaire. The questionnaire is conducted to 30 experts with over seven years of interior design teaching experiences to discuss influences these indicators have on students' creativity. Influences are classified from 1 to 10 , bigger number indicates higher influence to the students. The study collects 30 valid questionnaires in total. KolmogorovSmirnov test is used to examine its goodness of fit (K-S test is suitable for small sample examination, while the exam efficiency is better as well) to see the expert opinions belongs to normal distribution or non-normal distribution. This is followed by Fuzzy Delphi Method to filter its question item selection. Based on Center of Gravity (COG) by Klir \& Yuan to evaluate and filter all indicators [20]. With COG formula: $\mathrm{S}_{\mathrm{k}}=\left(\mathrm{a}_{\mathrm{k}}+\mathrm{b}_{\mathrm{k}}+\mathrm{c}_{\mathrm{k}}\right) / 3$ (fuzzy number $=$ (maximum value + minimum value + geometric mean) /3), the fuzzy number filter value the study gets is 7.27. Question items with lower filter value than 7.27 are eliminated, while the rest are considered to be the essential and valid indicators. These indicators can be used as references for systematic creativity development research.

\section{Research Results and Conclusions}

Followings are the analysis results of the normal parameter, K-S test, and fuzzy number filter value of the 130 indicators under the 10 dimension. 
Table 1. Results of the normal parameter, $K$-S test, and fuzzy number filter value of the 130 indicators.

\begin{tabular}{|c|c|c|c|c|c|c|c|c|c|}
\hline \multirow[b]{2}{*}{ Dimension } & \multirow{2}{*}{\multicolumn{2}{|c|}{ Indicator }} & \multicolumn{2}{|c|}{ Normal Parameter } & \multirow[b]{2}{*}{$\begin{array}{l}\text { K-S } \\
\text { Test }\end{array}$} & \multirow{2}{*}{$\begin{array}{l}\text { Asymp Siga } \\
\text { (2-Tailed) }\end{array}$} & \multirow[b]{2}{*}{$\begin{array}{l}\text { Normal } \\
\text { Distribution }\end{array}$} & \multirow[b]{2}{*}{$\begin{array}{l}\text { Fuzzy } \\
\text { Number }\end{array}$} & \multirow{2}{*}{$\begin{array}{l}\text { Filter }^{\mathrm{B}} \\
(6.21)\end{array}$} \\
\hline & & & Average & $\begin{array}{l}\text { Standard } \\
\text { Deviation } \\
\end{array}$ & & & & & \\
\hline \multirow{34}{*}{$\begin{array}{l}\text { A } \\
\text { Personality }\end{array}$} & \multicolumn{9}{|c|}{ A-1 Optimistic And Outgoing } \\
\hline & A-1-1 & Outgoing personality and generous mind & 7.43 & 1.72 & 1.26 & 0.09 & & 6.14 & $x$ \\
\hline & A-1-2 & Passionate about innovation & 9.40 & 0.72 & 1.62 & 0.01 & $x$ & 8.80 & (2) \\
\hline & A-1-3 & Active in crowd & 7.07 & 1.41 & 0.96 & 0.32 & & 7.36 & () \\
\hline & A-1-4 & Enthusiastic about helping the crowd & 7.10 & 1.81 & 0.70 & 0.70 & & 7.03 & () \\
\hline & \multicolumn{9}{|c|}{ A-2 Independent And Unique } \\
\hline & A-2-1 & Prefer acting alone or independently & 5.33 & 1.63 & 0.99 & 0.99 & & 5.11 & $x$ \\
\hline & A-2-2 & Intend to be different & 6.77 & 1.87 & 0.98 & 0.98 & & 6.26 & (2) \\
\hline & A-2-3 & Unique and unconventional thinking & 8.67 & 0.80 & 1.44 & 1.44 & $x$ & 8.56 & (a) \\
\hline & A-2-4 & True to personal style & 7.50 & 1.57 & 1.14 & 1.14 & & 7.17 & (0) \\
\hline & \multicolumn{9}{|c|}{ A-3 Diverse Interests } \\
\hline & A-3-1 & Love nature and outdoor goer & 6.67 & 1.56 & 0.92 & 0.37 & & 6.56 & (a) \\
\hline & A-3-2 & Exhibition fan & 8.73 & 1.20 & 1.21 & 0.11 & & 7.91 & () \\
\hline & A-3-3 & Enjoy traveling and adventure & 8.73 & 1.28 & 1.73 & 0.01 & $x$ & 7.91 & (a) \\
\hline & A-3-4 & Keep up with the latest trend & 8.77 & 1.01 & 1.42 & 0.04 & $x$ & 8.26 & () \\
\hline & $A-3-5$ & Extensive reading & 9.07 & 0.98 & 1.26 & 0.08 & & 8.36 & () \\
\hline & A-3-6 & Love to image & 9.10 & 0.84 & 1.57 & 0.01 & $x$ & 8.70 & () \\
\hline & A-3-7 & Love all sorts of arts & 8.97 & 0.89 & 1.36 & 0.05 & & 8.66 & () \\
\hline & \multicolumn{9}{|c|}{ A-4 Willing To Try Everything } \\
\hline & A-4-1 & Willing to try new things & 9.03 & 1.03 & 1.23 & 0.10 & & 8.34 & () \\
\hline & A-4-2 & Be adventurous and experimental & 8.97 & 1.30 & 1.39 & 0.04 & $x$ & 7.99 & (0) \\
\hline & A-4-3 & Dare to challenge & 8.20 & 1.37 & 1.14 & 0.15 & & 7.77 & (a) \\
\hline & A-4-4 & Thinking outside the box & 8.57 & 1.07 & 1.22 & 0.10 & & 8.19 & (a) \\
\hline & \multicolumn{9}{|c|}{ A-5 Self-Expression } \\
\hline & A-5-1 & Unyielding and emulative & 7.00 & 1.66 & 0.88 & 0.43 & & 6.33 & (0) \\
\hline & $A-5-2$ & Confident and dare to express & 8.23 & 1.30 & 0.85 & 0.47 & & 7.74 & (a) \\
\hline & $A-5-3$ & High self-discipline & 8.30 & 1.37 & 1.35 & 0.05 & & 7.77 & () \\
\hline & A-5-4 & Strive for efficiency & 7.23 & 1.91 & 0.86 & 0.46 & & 6.67 & () \\
\hline & A-5-5 & Perfectionism & 7.53 & 1.61 & 1.54 & 0.02 & $x$ & 7.18 & (0) \\
\hline & A-6 Dili & ent And Active & & & & & & & \\
\hline & A-6-1 & Hard-working attitude & 8.20 & 1.67 & 1.02 & 0.25 & & 7.07 & (a) \\
\hline & A-6-2 & Positive and active & 8.57 & 1.41 & 1.21 & 0.11 & & 7.86 & () \\
\hline & A-6-3 & Serious and pragmatic & 7.67 & 1.52 & 0.97 & 0.30 & & 7.22 & (0) \\
\hline & A-6-4 & Concentrate on work & 8.03 & 1.59 & 1.23 & 0.10 & & 7.34 & (a) \\
\hline & B-1 Inte & nal Motivation & & & & & & & \\
\hline & B-1-1 & Self-discipline and expectation & 8.33 & 1.32 & 1.24 & 0.09 & & 8.11 & () \\
\hline & B-1-2 & Prove one's own ability & 7.93 & 1.48 & 0.74 & 0.64 & & 7.64 & () \\
\hline & B-1-3 & Promote self-positioning & 7.87 & 1.43 & 0.88 & 0.42 & & 7.62 & () \\
\hline B & B-1-4 & $\begin{array}{l}\text { Strive for honor and sense of } \\
\text { achievement }\end{array}$ & 8.03 & 1.45 & 0.86 & 0.45 & & 7.68 & (0) \\
\hline Motivation & B-2 Exte & nal Motivation & & & & & & & \\
\hline & B-2-1 & Earn other's recognition & 7.63 & 1.16 & 0.95 & 0.32 & & 7.88 & () \\
\hline & B-2-2 & Gain rewards & 6.53 & 0.86 & 1.49 & 0.02 & $x$ & 6.51 & () \\
\hline & B-2-3 & Make a name for oneself & 6.83 & 1.15 & 0.98 & 0.29 & & 6.51 & () \\
\hline & B-2-4 & Altruism & 6.27 & 1.91 & 0.82 & 0.51 & & 6.09 & $x$ \\
\hline & B-2-5 & Teacher's inspiration and encouragement & 7.50 & 1.63 & 0.84 & 0.48 & & 6.83 & () \\
\hline & C-1 Self & learning & & & & & & & \\
\hline & C-1-1 & Active learning & 8.87 & 0.94 & 1.41 & 0.04 & $x$ & 8.62 & (2) \\
\hline & C-1-2 & Innumerable dabbling & 8.90 & 0.80 & 1.37 & 0.05 & & 8.63 & (a) \\
\hline & $\mathrm{C}-1-3$ & Comprehensive mastery & 8.87 & 1.17 & 1.28 & 0.07 & & 8.62 & () \\
\hline & C-1-4 & Continuous concentration & 8.80 & 0.96 & 1.18 & 0.12 & & 8.60 & () \\
\hline & $C-1-5$ & Knowledge accumulation & 8.73 & 0.87 & 1.21 & 0.11 & & 8.58 & (a) \\
\hline $\mathrm{C}$ & C-2 Flex & ble Conversion & & & & & & & \\
\hline Ability & $C-2-1$ & Knowledge introspection conversion & 8.77 & 0.90 & 1.48 & 0.03 & $x$ & 8.59 & (0) \\
\hline & $\mathrm{C}-2-2$ & Integrated and comprehensive proposal & 8.37 & 1.00 & 1.11 & 0.17 & & 8.46 & (a) \\
\hline & $\mathrm{C}-2-3$ & Accurate execution and plan & 8.17 & 1.23 & 0.84 & 0.48 & & 8.06 & () \\
\hline & $\mathrm{C}-2-4$ & Clear and complete expression & 8.57 & 1.14 & 1.00 & 0.27 & & 8.19 & () \\
\hline & C-3 Prot & lem-Solving & & & & & & & \\
\hline & C-3-1-1 & Finding problem & 8.47 & 1.14 & 1.17 & 0.13 & & 8.16 & (0) \\
\hline & $\mathrm{C}-3-2$ & Understanding problem & 8.37 & 1.03 & 1.08 & 0.20 & & 8.12 & (2) \\
\hline
\end{tabular}




\begin{tabular}{|c|c|c|c|c|c|c|c|c|c|}
\hline \multirow{5}{*}{ Dimension } & \multirow{2}{*}{\multicolumn{2}{|c|}{ Indicator }} & \multicolumn{2}{|c|}{ Normal Parameter } & \multirow{2}{*}{$\begin{array}{l}\text { K-S } \\
\text { Test }\end{array}$} & \multirow{2}{*}{$\begin{array}{l}\text { Asymp Siga }^{\mathrm{a}} \\
\text { (2-Tailed) }\end{array}$} & \multirow{2}{*}{$\begin{array}{l}\text { Normal } \\
\text { Distribution }\end{array}$} & \multirow{2}{*}{$\begin{array}{l}\text { Fuzzy } \\
\text { Number }\end{array}$} & \multirow{2}{*}{$\begin{array}{l}\text { Filter }^{\mathrm{B}} \\
(6.21)\end{array}$} \\
\hline & & & Average & $\begin{array}{l}\text { Standard } \\
\text { Deviation } \\
\end{array}$ & & & & & \\
\hline & $\mathrm{C}-3-3$ & Knowing solution & 8.63 & 1.13 & 1.06 & 0.21 & & 8.21 & (0) \\
\hline & C-3-4 & Problem solving & 8.73 & 1.08 & 1.26 & 0.08 & & 8.24 & ()) \\
\hline & $\mathrm{C}-3-5$ & $\begin{array}{l}\text { Problem solving experience } \\
\text { accumulation }\end{array}$ & 8.77 & 0.94 & 1.27 & 0.08 & & 8.59 & () \\
\hline \multirow{18}{*}{$\begin{array}{l}\text { D } \\
\text { Knowledge }\end{array}$} & \multicolumn{2}{|c|}{ D-1 General Knowledge } & & & & & & & \\
\hline & D-1-1 & General discipline knowledge & 7.10 & 1.90 & 1.00 & 0.27 & & 6.03 & $x$ \\
\hline & D-1-2 & Humanities and social science knowledge & 7.80 & 1.75 & 1.34 & 0.05 & & 6.60 & () \\
\hline & D-1-3 & $\begin{array}{l}\text { Design theory introduction and } \\
\text { knowledge }\end{array}$ & 8.53 & 1.41 & 1.08 & 0.20 & & 7.84 & (2) \\
\hline & D-1-4 & Architecture related basic knowledge & 8.23 & 1.55 & 1.13 & 0.15 & & 7.08 & (0) \\
\hline & D-1-5 & Interdisciplinary knowledge & 8.50 & 1.17 & 1.09 & 0.18 & & 7.83 & (0) \\
\hline & \multicolumn{2}{|c|}{ D-2 Professional Knowledge } & & & & & & & \\
\hline & D-2-1 & Basic interior design knowledge & 8.30 & 1.39 & 0.87 & 0.43 & & 7.77 & (2) \\
\hline & D-2-2 & Execution and method of interior design & 7.87 & 1.55 & 1.10 & 0.18 & & 7.29 & (0) \\
\hline & D-2-3 & Environment, material, and method & 7.90 & 1.47 & 1.06 & 0.21 & & 6.97 & ()) \\
\hline & $\begin{array}{l}\text { D-2- } \\
\text { 4DD-2-5 }\end{array}$ & $\begin{array}{l}\text { Design presentation and communication } \\
\text { skills }\end{array}$ & 8.67 & 1.03 & 1.06 & 0.21 & & 8.22 & (2) \\
\hline & D-2-5 & Relative regulations and contracts & 6.80 & 1.63 & 1.18 & 0.12 & & 6.27 & (2) \\
\hline & D-2-6 & $\begin{array}{l}\text { Professional practice ethics and } \\
\text { management }\end{array}$ & 6.93 & 1.98 & 0.99 & 0.28 & & 6.31 & (2) \\
\hline & D-2-7 & Aesthetics, sustainability, and innovation & 8.77 & 1.28 & 1.13 & 0.16 & & 7.92 & (2) \\
\hline & \multicolumn{2}{|c|}{ D-3 Nimble Knowledge } & & & & & & & \\
\hline & D-3-1 & Multi-collecting of data and information & 8.50 & 1.07 & 0.98 & 0.29 & & 8.17 & () \\
\hline & D-3-2 & Extensively gaining knowledge & 8.77 & 0.97 & 1.62 & 0.01 & $x$ & 8.26 & (2) \\
\hline & D-3-3 & Nimble usage of knowledge & 9.10 & 0.66 & 1.61 & 0.01 & $x$ & 9.03 & (2) \\
\hline \multirow{13}{*}{$\begin{array}{l}\text { E } \\
\text { Thinking }\end{array}$} & \multicolumn{2}{|c|}{ E-1 Thinking Method } & & & & & & & \\
\hline & E-1-1 & Illusory imagination & 8.00 & 1.34 & 0.94 & 0.33 & & 8.00 & (2) \\
\hline & E-1-2 & Multiple thinking & 8.67 & 0.99 & 1.63 & 0.01 & $x$ & 8.22 & (2) \\
\hline & E-1-3 & Independent thinking ability & 8.63 & 0.76 & 1.44 & 0.03 & $\times$ & 8.54 & ()) \\
\hline & E-1-4 & Reactive and quick response & 9.03 & 0.81 & 1.74 & 0.00 & $x$ & 8.68 & ()) \\
\hline & \multicolumn{2}{|c|}{ E-2 Logic Thinking } & & & & & & & \\
\hline & E-2-1 & Rational problem analyzing & 7.67 & 1.32 & 1.09 & 0.18 & & 7.22 & (0) \\
\hline & E-2-2 & Straight logical thinking & 8.33 & 0.96 & 1.26 & 0.08 & & 8.11 & (2) \\
\hline & E-2-3 & Convergent thinking & 7.73 & 1.36 & 1.16 & 0.14 & & 6.91 & (0) \\
\hline & \multicolumn{2}{|c|}{ E-3 Horizontal Thinking } & & & & & & & \\
\hline & E-3-1 & Divergent thinking & 8.17 & 1.18 & 1.33 & 0.06 & & 7.72 & (2) \\
\hline & E-3-2 & Keen to original ideas & 8.27 & 1.48 & 1.25 & 0.09 & & 7.09 & ()) \\
\hline & E-3-3 & Comprehensive views to question & 8.57 & 1.07 & 1.59 & 0.01 & $x$ & 8.19 & ()) \\
\hline & F-1 Famil & y Education & & & & & & & \\
\hline & F-1-1 & Open and liberal family education & 7.93 & 1.66 & 1.00 & 0.27 & & 6.98 & (0) \\
\hline & F-1-2 & Value and personality upbringing & 7.97 & 1.33 & 1.15 & 0.14 & & 7.66 & (2) \\
\hline & F-1-3 & Impressive family living experience & 7.93 & 1.57 & 0.83 & 0.50 & & 6.98 & ()) \\
\hline $\begin{array}{l}\text { F } \\
\text { Family }\end{array}$ & F-1-4 & $\begin{array}{l}\text { Parent education and soci-economic } \\
\text { background }\end{array}$ & 6.80 & 1.85 & 0.72 & 0.68 & & 5.93 & $x$ \\
\hline & F-2 Supp & ort And Encouragement & & & & & & & \\
\hline & F-2-1 & Family support and encouragement & 7.53 & 1.46 & 1.05 & 0.22 & & 7.51 & (0) \\
\hline & $\mathrm{F}-2-2$ & Family assistance & 7.13 & 1.48 & 0.90 & 0.39 & & 6.38 & ()) \\
\hline & $\mathrm{F}-2-3$ & Family guidance and suggestions & 6.23 & 1.63 & 0.96 & 0.32 & & 6.08 & $x$ \\
\hline & G-1 Passi & ng On Knowledge & & & & & & & \\
\hline & G-1-1 & Curriculum design fits professional needs & 8.30 & 1.18 & 1.09 & 0.18 & & 7.77 & (2) \\
\hline & G-1-2 & $\begin{array}{l}\text { Curriculum content is both theoretical } \\
\text { and practical }\end{array}$ & 8.10 & 1.32 & 1.01 & 0.25 & & 7.70 & (0) \\
\hline & G-1-3 & Teachers impart all knowledge & 8.37 & 1.10 & 1.11 & 0.17 & & 8.12 & (2) \\
\hline & G-1-4 & $\begin{array}{l}\text { Teachers are by example and precept } \\
\text { both }\end{array}$ & 8.17 & 1.15 & 1.28 & 0.08 & & 8.06 & (2) \\
\hline G & G-2 Hard & ware Facilities & & & & & & & \\
\hline & G-2-1 & Resourceful campus environment & 7.60 & 1.63 & 0.94 & 0.34 & & 6.87 & (0) \\
\hline & G-2-2 & Well-equipped facilities on campus & 7.57 & 1.41 & 0.98 & 0.29 & & 7.52 & (0) \\
\hline & G-2-3 & Students utilize every resource easily & 8.40 & 1.43 & 1.07 & 0.20 & & 7.80 & ()) \\
\hline & G-2-4 & $\begin{array}{l}\text { Resource is under good management and } \\
\text { maintenance }\end{array}$ & 7.53 & 1.43 & 1.17 & 0.13 & & 7.18 & \\
\hline & G-3 Cam & us Atmosphere & & & & & & & \\
\hline & G-3-1 & Open and liberal education & 8.43 & 0.97 & 1.39 & 0.04 & $x$ & 8.14 & () \\
\hline
\end{tabular}




\begin{tabular}{|c|c|c|c|c|c|c|c|c|c|}
\hline \multirow[b]{2}{*}{ Dimension } & \multirow{2}{*}{\multicolumn{2}{|c|}{ Indicator }} & \multicolumn{2}{|c|}{ Normal Parameter } & \multirow{2}{*}{$\begin{array}{l}\text { K-S } \\
\text { Test }\end{array}$} & \multirow{2}{*}{$\begin{array}{l}\text { Asymp Siga }^{a} \\
\text { (2-Tailed) }\end{array}$} & \multirow{2}{*}{$\begin{array}{l}\text { Normal } \\
\text { Distribution }\end{array}$} & \multirow{2}{*}{$\begin{array}{l}\text { Fuzzy } \\
\text { Number }\end{array}$} & \multirow{2}{*}{$\begin{array}{l}\text { Filter }^{\mathrm{B}} \\
(6.21)\end{array}$} \\
\hline & & & Average & $\begin{array}{l}\text { Standard } \\
\text { Deviation } \\
\end{array}$ & & & & & \\
\hline \multirow{20}{*}{$\begin{array}{l}\mathrm{H} \\
\text { Student club }\end{array}$} & G-3-2 & $\begin{array}{l}\text { Encourage creative and independent } \\
\text { thinking }\end{array}$ & 9.00 & 0.87 & 1.64 & 0.01 & $x$ & 8.33 & (2) \\
\hline & G-3-3 & Peer inspiration and learning & 8.73 & 0.94 & 1.52 & 0.02 & \multirow[t]{2}{*}{$x$} & 8.58 & (2) \\
\hline & G-3-4 & Respect from faculty to students & 8.07 & 1.31 & 1.07 & 0.20 & & 7.69 & (2) \\
\hline & \multicolumn{9}{|c|}{ H-1 Interpersonal Communication } \\
\hline & H-1-1 & $\begin{array}{l}\text { Cultivation of interpersonal } \\
\text { communication skills }\end{array}$ & 7.50 & 1.33 & 1.21 & 0.11 & & 7.50 & (2) \\
\hline & H-1-2 & Building coordination ability & 8.00 & 1.11 & 1.28 & 0.08 & & 7.67 & ()) \\
\hline & $\mathrm{H}-1-3$ & Establishment of good relationship & 7.67 & 1.12 & 1.19 & 012 & & 7.56 & (2) \\
\hline & $\mathrm{H}-1-4$ & Respects different viewpoints & 8.23 & 1.04 & 1.11 & 0.17 & & 8.08 & ()) \\
\hline & \multicolumn{9}{|c|}{ H-2 Group Interaction } \\
\hline & $\mathrm{H}-2-1$ & Invincible teamwork & 8.13 & 1.48 & 1.08 & 0.19 & & 7.71 & (2) \\
\hline & $\mathrm{H}-2-2$ & Team spirits and silent understanding & 7.97 & 1.30 & 1.15 & 0.14 & & 7.66 & () \\
\hline & $\mathrm{H}-2-3$ & $\begin{array}{l}\text { Learn from each other and co- } \\
\text { competition }\end{array}$ & 7.37 & 0.96 & 1.36 & 0.05 & & 8.07 & (0) \\
\hline & $\mathrm{H}-2-4$ & Discussion and inspiration & 7.77 & 1.09 & 1.14 & 0.15 & & 8.23 & () \\
\hline & \multicolumn{9}{|c|}{ H-3 Organization Composition } \\
\hline & $\mathrm{H}-3-1$ & Organizational mission and goal & 6.93 & 1.57 & 0.76 & 0.61 & & 6.64 & (2) \\
\hline & $\mathrm{H}-3-2$ & Characteristics of team member & 7.67 & 1.35 & 1.08 & 0.19 & & 7.56 & (2) \\
\hline & $\mathrm{H}-3-3$ & Composition of team member & 7.37 & 1.40 & 1.14 & 0.15 & & 7.12 & (2) \\
\hline & $\mathrm{H}-3-4$ & Teamwork and collaboration & 7.77 & 1.22 & 0.96 & 0.31 & & 7.59 & ()) \\
\hline & \multicolumn{9}{|c|}{ I-1 Social Agendas } \\
\hline & $\mathrm{I}-1-1$ & Reflect essence and features of time & 7.87 & 1.28 & 1.14 & 0.15 & & 7.62 & ()) \\
\hline \multirow{13}{*}{$\begin{array}{l}\text { I } \\
\text { Criteria }\end{array}$} & $\mathrm{I}-1-2$ & Highlight and pass on cultural attributes & 7.90 & 1.54 & 1.05 & 0.22 & & 6.97 & (2) \\
\hline & $\mathrm{I}-1-3$ & Create eye-catching topics & 8.03 & 1.38 & 1.41 & 0.04 & $x$ & 7.68 & () \\
\hline & $\mathrm{I}-1-4$ & Judgment to social phenomenon & 7.77 & 1.76 & 1.39 & 0.04 & $x$ & 6.59 & (2) \\
\hline & \multicolumn{9}{|c|}{ I-2 Performance Outcome } \\
\hline & $\mathrm{I}-2-1$ & Excellent expression and presentation & 8.30 & 1.09 & 1.14 & 0.15 & & 8.10 & (2) \\
\hline & $\mathrm{I}-2-2$ & Professional standard and attributes & 8.43 & 1.10 & 1.02 & 0.25 & & 8.14 & () \\
\hline & $\mathrm{I}-2-3$ & Innovating content and approach & 8.70 & 1.09 & 1.14 & 0.15 & & 8.23 & (2) \\
\hline & $\mathrm{I}-2-4$ & Complete and pertinent work & 8.63 & 1.00 & 1.11 & 0.17 & & 8.54 & () \\
\hline & \multicolumn{9}{|c|}{ I-3 Demand Satisfaction } \\
\hline & $\mathrm{I}-3-1$ & Results satisfy users' demand & 8.17 & 1.49 & 1.03 & 0.24 & & 7.39 & (a) \\
\hline & $\mathrm{I}-3-2$ & Equally attractive and practical & 8.07 & 1.44 & 1.33 & 0.06 & & 7.36 & () \\
\hline & $\mathrm{I}-3-3$ & Distinctive and appropriate theme style & 8.13 & 1.28 & 1.23 & 0.10 & & 7.71 & (2) \\
\hline & $\mathrm{I}-3-4$ & Originality and uniqueness & 9.00 & 1.02 & 1.28 & 0.08 & & 8.33 & (a) \\
\hline \multirow{14}{*}{$\begin{array}{l}\text { J } \\
\text { Stakeholder }\end{array}$} & \multicolumn{9}{|c|}{ J-1 Instructor } \\
\hline & $\mathrm{J}-1-1$ & Inspire students to be creative & 8.97 & 0.81 & 1.37 & 0.05 & & 8.66 & (2) \\
\hline & $\mathrm{J}-1-2$ & Point out the potential problem & 8.43 & 1.01 & 1.35 & 0.05 & & 8.14 & (2) \\
\hline & $\mathrm{J}-1-3$ & $\begin{array}{l}\text { Allow students to fully express } \\
\text { themselves }\end{array}$ & 8.33 & 1.45 & 1.14 & 0.15 & & 7.78 & (2) \\
\hline & $\mathrm{J}-1-4$ & Supervision and guidance & 8.20 & 1.21 & 1.34 & 0.05 & & 7.73 & ()) \\
\hline & $\mathrm{J}-1-5$ & Encouraging and supportive attitude & 8.53 & 1.22 & 1.00 & 0.27 & & 8.18 & (2) \\
\hline & \multicolumn{9}{|c|}{ J-2 Scholar And Expert } \\
\hline & $\mathrm{J}-2-1$ & Professional advice from experts & 8.10 & 1.16 & 1.18 & 0.12 & & 7.70 & (2) \\
\hline & $\mathrm{J}-2-2$ & Practitioners offer practical suggestions & 7.87 & 1.14 & 1.35 & 0.05 & & 7.29 & ()) \\
\hline & $\mathrm{J}-2-3$ & $\begin{array}{l}\text { Scholars and experts provide necessary } \\
\text { assistance }\end{array}$ & 8.10 & 0.99 & 1.24 & 0.09 & & 8.03 & (2) \\
\hline & J-3 User & & & & & & & & \\
\hline & $\mathrm{J}-3-1$ & $\begin{array}{l}\text { Users' behavior observation and } \\
\text { stimulation }\end{array}$ & 8.43 & 1.14 & 1.18 & 0.12 & & 8.14 & (a) \\
\hline & $\mathrm{J}-3-2$ & Analysis of users' demand & 8.37 & 0.96 & 1.54 & 0.02 & $\times$ & 8.46 & (2) \\
\hline & $\mathrm{J}-3-3$ & Discussion with potential users & 8.70 & 0.99 & 1.25 & 0.09 & & 8.57 & ()) \\
\hline
\end{tabular}

${ }^{\mathrm{A}} \mathrm{K}-\mathrm{S}$ test is used to examine the normal distribution of expert opinion, asymptotic significance (two-tailed). H0 is rejected at significance level lower than .05, showing significant observed frequency and theoretical frequency. The result indicates that expert opinion is not normally distributed. $\times$ is indicates non normal distribution indicator (expert opinions are consistent).

${ }^{\mathrm{B}}$ Filter value of fuzzy number is set up by fuzzy number of all indicators. It is set at 6.21 (minimum value is 1 , maximum value is 10 , and the geometric mean of all fuzzy number is 7.63). (O) indicates its fuzzy number is higher than filter value, while $\times$ indicates deleted indicators, and the entire row is marked in grey 
Table 2. Analysis of question items of 10 dimensions.

\begin{tabular}{llllll}
\hline Dimension & Original question items & Deleted question items & Question items after deletion & Fuzzy number & Ranking \\
\hline A. Personality & 28 & 2 & 26 & 8.09 & 3 \\
B. Motivation & 9 & 1 & 8 & 7.79 & 8.41 \\
C. Ability & 14 & 0 & 14 & 8.04 & 8 \\
D. Knowledge & 15 & 1 & 14 & 8.21 & 5 \\
E. Thinking & 10 & 0 & 10 & 7.59 & 2 \\
F. Family & 7 & 2 & 5 & 7.97 & 10 \\
G. School & 12 & 0 & 12 & 7.76 & 6 \\
H. Student club & 12 & 0 & 12 & 7.91 & 9 \\
I. Criteria & 12 & 0 & 12 & 8.06 & 7 \\
J. Stakeholder & 11 & 0 & 11 & 124 & 4 \\
Total & 130 & 6 & & \\
\hline
\end{tabular}

After the analysis, six deleted indicators are, A-1-1 (outgoing personality and generous mind), A-2-1 (prefer acting alone or independently), B-2-4 (Altruism), D-1-1 (general discipline knowledge), F-1-4 (parent education and soci-economic background), and F-2-3 (family guidance and suggestions), which narrows the questionnaire down to 124 indicators (from 130). The Cronbach's $\alpha$ merely drops down to 0.959 from 0.966 . The top three dimensions that affect interior design students' creativity are ability, thinking, and personality, while the bottom three are family, student club, and motivation. The three most influential indicators in descending order are D-3-3 (nimble usage of knowledge), A1-2 (passionate about innovation), and A-3-6 (love to image). This study adopts Csikszentmihalyi's systems model of creativity, which is an unverified conceptual model even till now. The study results can be used to examine its viewpoints, as well as to establish a systematical creativity scale that fits interior design business. If a mega scale norm can be established in the future, the interior design creativity scale will be normreferenced for further research study. These study results would be beneficial to creativity development and cultivation on college education for interior design students.

\section{References}

[1] Amabile, T. M. (1983). The Social Psychology of Creativity. NY: Springer-Verlag.

[2] Csikszentmihalyi, M. (1988). Society, culture, and person: A systems view of creativity. In R. J. Sternberg (Ed.), The Nature of Creativity: Contemporary Psychological Perspectives (pp. 325-339). NY: Cambridge University Press.

[3] Sternberg, R. J., \& Lubart, T. I. (1999). The concept of creativity: Prospects and paradigms. In R. J. Sternberg (Ed.), Handbook of Creativity.(pp. 3-15). UK: Cambridge University Press.

[4] Sternberg, R. J., \& Lubart, T. L. (1996). Investing in creativity. American Psychologist, 51(7): 677-688.

[5] Yang, K. Y. (2001) The introduction of the meaning and influential factors of creativity, Science Education Monthly, 239: 3-12.

[6] Yeh, Y. C. (2000) "The Ecological Systems Model of Creativity Development" and its Contest Interpretation in the Fields of Technology and Information, Bulletin of Educational Psychology, 32(1): 95-122.

[7] Shieh, P. F. Hung, J. F. (2006), The research of scientific creativity assessment from the aspect of confluence approach, Science Education Monthly, 291: 11-23.

[8] Niu, W. (2007). Individual and environmental influences on Chinese student creativity. Journal of Creative Behavior, 41(3):151-175.

[9] Csikszentimihalyi, M. (1999). Implications of a systems perspective for the study of creativity. In R. J. Strenberg (Ed.), Handbook of Creativity (pp. 313-335). NY: Cambridge University Press.

[10] Mao, L. W., Kuo, Y. Y. Chen, L. A. and Lin, S. T. (2000) Research of Creativity. Taipei: Psychological Publishing Co., Ltd.

[11] Chen, J. H. (2015) The Study on Students' Creativity from Business Innovation Field of Technological and Vocational Education: The Creativity Process Exploration, Creativity Indicators Construction and Systemic Creativity Scale Establishment. Project granted by National Science Council, the Executive Yuan. Grant No. NSC 99-2511-S-122-001 (in Chinese).

[12] Runco, M. A., Nemiro, J., \& Wallberg, H. J. (1998). Personal explicit theories of creativity. Journal of Creative Behavior, 32: 1-17.

[13] Torrance, E. P. (1988). The nature of creativity as manifest in its testing. In R. J. Sternberg (Ed.), The Nature of Creativity: Contemporary Psychological Perspectives. (pp. 43-75). Cambridge University Press.

[14] Csikszentmihalyi, M. (1996). Creativity: Flow and the Psychology of Discovery and Invention. NY: Harper Collins Publishers.

[15] Csikszentmihalyi, M. (1990). The domain of creativity. In M. A. Runco \& R. S. Albert (Eds.). Theories of creativity (pp.190-212). London: Sage Publications 
[16] Gardner, H. (1993). Frames of Mind: The Theory of Multiple Intelligences. New York: Basic.

[17] Mayer, R. E. (1999). Fifty years of creativity research. In R. J. Sternberg (Ed.), Handbook of creativity (pp. 449-460). Cambridge, UK: Cambridge University Press.

[18] Ming-Hsiung Wu, Pei-Chun Shih, Hsin-Cheng Chen, KueiMiao Lin, Yu-Ju Cheng, Wang Yu (2012), Construction of systematic creativity indicators for undergraduate students in industrial technology field of technological and vocational education, in industrial technology field of technological and vocational education, 2012 4th International Conference on
Environmental Science and Information Application Technology Advances in Biomedical Engineering, Vol. 14.

[19] Chung, S. T. (2012) Creativity Research on Interior Design Students in Vocational Schools: Discussion on Process, Indicator Construction, and Systematical Creativity Scale Establishment II. Project granted by National Science Council, the Executive Yuan. Grant No. NSC 100-2511-S-003-006 (in Chinese).

[20] Klir, G. J., \& Yuan, B. (1995). Fuzzy Sets and Fuzzy LogicTheory and Application. NJ: Prentice-HallInc. 